\title{
Lincomycin Hydrochloride Anhydrous
}

National Cancer Institute

\section{Source}

National Cancer Institute. Lincomycin Hydrochloride Anhydrous. NCI Thesaurus. Code C90876.

The anhydrous hydrochloride salt form of lincomycin, a lincosamide antibiotic originally identified in actinomycete Streptomyces lincolnensis with activity ag ainst gram-positive cocci and anaerobic bacteria. 